\title{
Zur Entwicklung der Vena cardinalis posterior von Torpedo und deren Beziehungen zur Vorniere. \\ Von
}

J. Rückert, München.

Hierzu Tafel XXV und XXVI und 1 Textfigur.

Die nachstehende Untersuchung, welche sich mit der Entstehung der Kardinalvene im Vornierengebiet von Torpedo beschaftigt, schliesst sich an meine vorangehende Arbeit "Über die Glomeruli der Vorniere von Torpedo und deren arterielle Gefässe “ (Arch. f. mikrosk. Anat., Bd. 89, Heft 4, 1916) an. Es wird daher in ihr wiederholt auf diese Publikation Bezug genommen. Wenn die Entstehung der Venen der Vornierengegend hier gesondert von derjenigen der Arterien dargestellt wird, so mag dies in dem Umstande seine Rechtfertigung finden, dass bei Torpedo diese beiderlei Gefässe nicht gleichzeitig auftreten, sondern die Venen merklich spater als die Arterien.

\section{Selachierliteratur.}

In der Literatur finden sich über die erste Entstehung der Kardinalvenen bei Elasmobranchiern folgende Angaben. C. Rabl teilt in seiner Schrift „Über die Entwicklung des Venensystems der Selachier" (1892) mit, dass die Anlagen der V. cardinalis posterior bei Pristiurusembryonen von 60 und 70 Urwirbeln noch sehr kurz sind und kaum über die Leberanlage hinausreichen, während die vordere Kardinalvene gut entwickelt und vielleicht schon bei Embryonen von 56 Urwirbeln vorhanden ist. Über die Art ihrer Entstehung äussert er sich nicht bestimmt, doch hält er es für möglich, dass sie nicht vom Duct. Cuvieri aussprosst; sondern durch Vereinigung der venösen Schenkel der von den Aorten entspringenden Intersegmentalarterien ( Interprotovertebralarterien" der Autoren) entsteht. Die V. cardinalis posterior liegt dann in der hinteren Rumpfbălfte seitlich von dem in Bildung begriffenen Interrenalkörper, weiter vorn neben der Aorta und dabei stets dorsal von den Urnierenkanälchen“. Im folgenden Jahre beschreibt dann C. K. Hoffmann, dem die Rablsche 
Publikation nicht zugänglich war, die Kardinalvenenanlagen zuerst (1893a) als "Ausstülpungen des Sinus venosus" und darauf (1893 b) als segmentale Ausbuchtungen der Aorta. Die letztere Entstehungsweise der Kardinalvene ist auch für andere Objekte, so in neuerer Zeit für das Hühnchen von Grafe (1905) und Evans (1909) angegeben worden. Auch $\mathrm{H}$ of $\mathrm{fmann}$ lässt die hintere Kardinalvene später entstehen als die vordere.

\section{Eigene Befunde über die erste Anlage der hinteren Kardinalvene.}

Meine eigenen Untersuchungen über die Entwicklung der hinteren Kardinalvene betrachte ich keineswegs als erschöpfende, es kommt mir nur darauf an, die Entstehung der Vene im Bereich der Vorniere zu verfolgen und einen auffallenden Unterschied in der Entwicklung dieses Stückes und des übrigen Rumpfabschnittes klarzustellen. Da ich auf die Entstehungsweise des Duct. Cuvieri und des anschliessenden, bis zum Vorderende der Vorniere reichenden kurzen Venenabschnittes im folgenden nicht zurückkomme, erwähne ich hier nur kurz, dass auch ich ein Auswachsen der Vene aus dem Duct. Cuvieri nicht gefunden habe und bestatige ferner die leicht festzustellende Tatsache, dass die hintere Kardinalvene später auftritt als die vordere. Dass ihre Anlage aus Ausbuchtungen der Aorta oder aus Aortenästen hervorgeht, ${ }^{1}$ ) trifft dagegen für Torpedo nicht $\mathrm{zu}$. Sie erscheint vielmehr wie diejenigen der übrigen grossen Gefäßstämme zuerst in Form einer Ansammlung von Gefässzellen, die auf einzelnen Querschnitten nur spärlich, oft nur in der Einzahl vorhanden sind, neben der schon bohlen Aorta und ohne Verbindung mit ihr, wie wir seben werden. Aus dieser Anlage gehen dann in der bekannten Weise Endothelröhrchen hervor, die aber gleichfalls nicht mit der Aorta in Verbindung stehen. Dagegen will ich nicht in Abrede stellen, dass ein Zusammenhang mit den Anlagen von Intersegmentalgefassen besteht, doch habe ich diesem Punkt keine besondere Aufmerksamkeit geschenkt. Ihre Lage zu den Urnierenkanälchen bzw. den Urwirbelstielen wird dagegen eingehend behandelt werden.

1) Das letztere ist nach neueren Angaben Möllendorfs (1911) bei Bombinator der Fall. Vgl. auch Grafe und Evans. 
Bei Torpedo tritt die Anlage der hinteren Kaldinalvene zuerst in der Vornierengegend und bald danach auf der kurzen Strecke zwischen dem Duct. Cuvieri und der Vorniere auf. Sie breitet sich langsam bis zum hinteren Ende der Vorniere aus und greift von da in del gleichen Richtung fortschreitend auf die weiter kaudal gelegenen 'Teile des Rumpfes über.

In der Vornielenregion, auf die ich mich zunächst beschränke, erscheint die Vene erst zu einer Zeit, in welcher die arteriellen Quergefasse, besonders die rasch vergänglichen linksseitigen, und die Glomerularanlagen schon wieder in Rückbildung begriffen sind. Infolgedessen ist ihr Verbaltnis $z$ den Glomeruli bei unserem Objekt viel schwieriger klalzustellen als bei den übrigen Anamniern, bei denen diese Gebilde eine vollkommenere Entwicklungsstufe erreichen und die Kardinalvene das Blut aus funktionierenden Glomerulis zurückführt.

Beginnen wir die Beschreibung mit dem Entwicklungsstadium, welches der voranstehenden Arbeit zugrunde liegt, in welchem also von fünf Visceraltaschen die zweite und dritte erötfinet ist und 39 Rumpfsomiten vorbanden sind. Bei dem einen der zwei quergeschnittenen Embryonen dieses Stadiums (Torp. 52) fand sich auf der rechten Seite die erste Spur der hinteren Kardinalvene in Gestalt eines sehr engen Endothelröhrchens, das medial dicht neben der Vornierenrinne, zwischen ihr und dem Urwirbelstiel bzw. dem Quergefäss liegt (siehe Rü ckert, 1916, Taf. XXIII, Fig. 20). Das junge Hohlgefăss dehnt sich nur über ein paar Schnitte aus, in einer Querschnittsebene, die dicht vor dem zweiten arteriellen Quergefäss liegt. Vor ihm und hinter ihm (1. c., Fig. 21) finden sich als solide Vorstufen des Venenrohrs nur ein kleines Zellenhäufchen oder vereinzelte Gefässzellen, die sich von den zwischen den Urwirbelstielen gelegenen Mesenchymzellen nicht immer mit Sicherheit unterscheiden lassen.

Auf der linken Seite des Embryo und an einer zweiten Querschnittserie des gleichen Stadiums (Torp. 54) fehlt noch jede Spur eines Hohlgefässes, hier sind nur die zuletzterwähnten zelligen Vorläufer der Anlage, und auch diese in geringerer Zahl, vorbanden.

Ebenso verhălt sich ein Embryo mit 41 und 42 Rumpfsomiten, eröffneter erster bis dritter und in Durchbruch begriffener 
vierter Visceraltasche. Bei diesem sowie bei den naichstfolgenden Embryonen sind die Glomeruli, besonders die linken. schon erheblich zurückgebildet.

Auch bei cinem Embryo mit 43-44 Rumptsomiten und fünf Visceraltaschen, von denen die erste bis vierte eröffnet sind, ist die Anlage der linken Kardinalvene noch auf der nämlichen sehr früben Stufe, während sie rechts in den Querschnittsebenen des ersten und dritten Quergefässes über je ein paar Schnitte die Form eines Endotlelröhrehens erreicht hat.

Bei einem gleichaltrigen Embryo dagegen erscheint die Vene auf der rechten Seite als Endothelrohr am vorderen Ende der Vorniere schon über eine etwas längere Strecke. Sie dehnt sich über den ganzen Zwischenraum zwischen dem ersten und zweiten Nephrotom aus und liegt hier (Taf. XXV Fig. 1) in einer Hachen, ventral vorgebuchteten Falte der dorsalen Leibeshöhlenwand, in welche dicht medial von ihr ein kurzer Aortenzipfèl, das Rudiment eines ersten Quergefasses (Q), vordringt. Die Peritonealfalte ist somit als eine rudimentäre Glomerularfalte anzusprechen, von der Art, wie sie für die linke Körperseite von mir ( $R$ ückert, 1916) dargestellt worden ist. Das neue ist nur, dass in ihr neben dem arteriellen jetzt auch ein venöses (iefäss liegt. Wie man das erstere als zuführendes. so darf mandas letztere a ls abführendes Glomerulargefäss betrachten.

Mit dem Auftreten des kaudal sich auschliessenden Crwirbelstiels (Nephrotoms Fig. $2 \mathrm{~N}$ ), dessen Hohlraum medial von der Falte gelegen. nach aufwärts und lateral vordringt, wird die letztere tiefer ausgeschnitten. Sie ragt jetzt in ventro-medianer Richtung in die Leibeshöhle vor. Auch ist sie mitsamt ibrer Vene durch den Urwirbelstiel nach abwärts verdrăngt worden, wie am deutlichsten aus der Lageveränderung des Gefüssquerschnittes zum dorsalen Darmrand oder zur ventralen Aortenwand ersichtlich wird.

Noch weiter kaudal (Fig. 3) tritt die herabgetretene Falte auf das dichte Mesenchymlager (Ml), welches das zur Aorta aufsteigende vordere Ende der Dotterarterie (Da) bedeckt, und verbindet sich mit ihm. Dabei geht ihr Peritonealüberzug in die den Mesenchymwulst überkleidende und durch ihn vom Darm abgehobene Splanchnopleura über, so dass die im Innern der Falte gelegene Vene direkt auf das Mesenchymlager gelangt. Dem 
rétzteren aber sitzen, da wo es dorsal bis an die Vorniere heranreicht, die Glomeruluszapfen auf, wie in der voranstehenden Arbeit gezeigt wurde.

Es geht also aus dem Gesagten hervor, dass an vorderen Ende der Forniere die frisch gebildete liardinalvene sich a $\mathrm{f}$ das Glomerulargebiet herabsenkt und zwar innerhalb einer Falte der dorsalen Peritonealwand, die als "Cölomstrang" (Fig. 3, St) den dorsalen Abschnitt der Leibeshöhle in ventromedianer und zugleich etwas kaudaler Richtung durchsetzend zur Mesenchymdecke der Dotterarterie zieht.

Bei anderen Embryonen, bei welchen der vordere Umfang der Dotterarterie etwas weiter kaudal emporsteigt, schlägt der Cölomstrang mehr die Richtung nach hinten ein und wird daher auf Querschnitten fast quer getroffen, wie aus der Abbildung eines gleiclı \%u besprechenden etwas älteren Embryos (Fig. 4, St) ersichtlich ist.

Den beschriebenen Cölomstrang fand ich schon bei einem 'Teil der jüngeren Embryonen, die noch keine Spur einer Kardinalvenenanlage aufweisen. Fr stellt zu jener Zeit einen soliden, d. h. gefasslosen Zellenzug dar. Erst wenn mit dem Auftreten der binteren Kardinalvene ein Endothelröhrchen in ihm erscheint, habe ich ihn konstant angetroffen. Von da an erbält er sich, auch nach dem Verlust des Gefässes, über eine lange Zeit des Embryonallebens.

Wir fahren in der Beschreibung unseres Stadiums fort. Nachdem die Kardinalvene in der geschilderten Weise aus ihrer Lage abgelenkt ist, tritt sie kaudal nach längerer Unterbrechung erst zu Beginn des zweiten, durchlaufenden, arteriellen Quergefässes der Vorniere wiederum, und zwar an ihrer alten Stelle neben der Aorta, als enges Endothelröhrchen über ein paar Schnitte anf.

Auf der linken Seite erscheint die Vene gegenüber der Fig. 1 ebenfalls zwischen zwei Urwirbelstielen, aber nur über wenig Schnitte. Sie liegt hier gleichfalls in einer Peritonealfalte. Diese schnürt sich jedoch links niemals : zu einem Strang ab, entsprechend dem Umstand, dass hier keine Dotterarterie gebildet wird, der sie zustreben könnte. Auch hier taucht sie hinter dem übernächsten Nephrotom nochmals auf, doch nur als solide Anlage. Aber diese liegt in der Basis einer schwachen Peritonealfalte, 
lateral neben einem Aortenast, ähnlich wie in Fig. 1 der rechten Seite, so dass man auch hier von einer Glomerulusfalte sprechen kann. Es folgen dann noch drei kräftig ausgebildete Aortenäste (Glomerulararterien) mit woblerhaltenen Glomerulusfalten, aber in deren Bereich ist die Vene noch nicht angelegt. Da zwei dieser Gefässe schon von der Aorta abgetrennt sind, kann man sie leicht mit der Anlage der Kardinalvene verwechseln und so zu der Annahme kommen, dass die letztere in Form von intermetameren Aortenbuchten entstehe. Vielleicht klärt sich der Irrtum C. K. Hoffmanns (s. S. 467) auf diese Weise auf.

Einen weiteren Fortschritt ibrer Langenentwicklung zeigt die hintere Kardinalvene bei einem nur wenig älteren Embryo, bei welchem die sechste Visceraltasche als schwache Ausbuchtung schon angelegt ist, aber nur die vier ersten Visceraltaschen, wie bei dem vorigen Embryo, eröffnet sind.

Über die linke Seite dieses Embryo können wir kur\% hiıweggehen. Die Vene dehnt sich hier über die vordere Hălfte des Vornierengebiets aus und zwar mit zweimaliger Unterbrechung an den Crwirbelstielen. Das erste Rohrstück gelangt beim Einschneiden des Urwirbelstiels, indem es solid und unfertig wird, in die gleiche Peritonealfalte, wie sie in Fig. 2 für die rechte Seite des vorigen Embryos abgebildet ist. Das zweite und dritte Venenstückchen liegen in gan $z$ Hachen, nur erst angedeuteten Falten. Das dritte hat zwar neben sich einen noch wohl erhaltenen Aortenast. doch kann man von einer "Glomerularfalte" hier nicht mehr sprechen. Erst am folgenden Aortenast ist eine solche noch vorhanden, hier ist aber die Vene noch nicht angelegt.

Auf der rechten Seite erstreckt sich die Vene kaudal bis über die Mitte der Vornierenregion hinaus. Sie ist noch unterbrochen und zwar wie bisher an den Stellen der Urwirbelstiele, wie aus dem nach einer graphischen Rekonstruktion hergestellten Übersichtsbild der Fig. $11 \mathrm{zu}$ erkennen ist. Die Vene ist hier schraffiert, die Urwirbelstiele sind mit schwarzem Farbenton angegeben.

Im Einzelnen verhält sie sich bei unserem -Embryo folgendermassen. Am vorderen, ganz Hachen und unbestimmten Ende der Vorniere.geht von der Stelle, wo die Vene liegt, eine Peritonéalfalte aus, die sich in den oben beschriebenen "Cölomstrang" (Fig. 4) fortsetzt. Dabei besteht gegenüber dem vorigen Embryo 
der nebensächliche Unterschied, dass die Vene nicht als Hohlgefäss in die Falte eintritt, sondern blind an ibr endet. Aber einige Zellen, die man als ihre Fortsetzung ansehen darf, gelangen in das Innere der Falte, und an deren Stelle erscheint dann im weiteren Verlauf des Stranges durch die Leibeshöhle das in Fig. 1 abgebildete Hohlgefăss, das sich zum Mesenchymlager der Dotterarterie herabsenkt. Dieses Gefäss ist in Fig. 11 unterhalb des ersten Urwirbelstieles zu sehen $\left(\mathrm{Cv}_{1}\right)$.

In den Zwischenräumen $z$ wischen diesem und dem folgenden Urwirbelstiele taucht nun die unterbrochene Kardinalvene $\left(\mathrm{C}_{2}\right.$ in Fig. 11) wieder an ihrem alten Platz, am ventro-lateralen Umfang der Aorta, auf (Fig. 4 (). Sie kommt auf den folgenden Schnitten dicht neben das erste arterielle Quergefilss zu liegen, das von der Aorta zur Dotterarterie durchläuft. Fig. 1 zeigt schon die Stelle, an welcher die Aorta sich zu diesem Quergefiss (Q) auszieht, im vorderen Anschnitt und gibt so eine Vorstellung von der Lage der Vene zur Arterie.

Durch den nächsten Urwirbelstiel (zweites Nephrotom) wird die Vene wieder mitsamt dem angrenzenden Leibeshöhlendach ventral verschoben und von der Aorta abgedrangt, ähnlich wie es in Fig. 5 für ein späteres Segment dargestellt ist (Fig. $11 \mathrm{CV}_{2}$ ). Alsdann endet sie wieder im Bereich desselben Urwirbelstiels. Hinter ihm tritt sie zum dritten Male auf (Fig. $11 \mathrm{C}_{3}$ ), kommt neben"das zweite arterielle Quergefäss und wird sodann durch das dritte Nephrotom wiederum ventral verdrängt (Fig. 11 (v). Diese Stelle wurde auf dem Querschnitt der Fig. 5 abgebildet; weil hier die Vene sich bezeichnenderweise ventral gegen das Mesenchymlager spitz auszieht, als ob sie in dasselbe einzudringen im Begriff stünde und weil am Mesenchymlager sich hier noch ein Glomeruluszapfen (GI) erhalten..hat. Nach einer kurzen Unterbrechung durch das dritte Nephrotom erscheint die Vene im Bereich des dritten und weitesten Quergefüsses nochmals auf ein paar Schnitte, zum vierten und letzten Male $\left(\mathrm{C}_{4}\right.$ in Fig. 11).

Wie hieraus ersichtlich, tritt die hintere Kardinalvene in der vorderen Halfte des Vornierengebietes intermetamer zwischen den Urwirbelstielen auf, um sich von diesen Stellen aus: noch ein längeres oder kürzeres Stück in das Bereich des nächstfolgenden Urwirbelstiels fortzusetzen, wobei sie von der; 
Aorta ventral abrückt und sich dem Glomerulusgebiet nähert. Hierbei zeigt sie nun in den einzelnen Segmenten ein verschiedenes Verbalten. Während sie nämlich am vordersten Urwirbelstiel der Region in eine ventral in die Leibeshöhle vorragende Peritonealfalte (Fig. 2) gerät, die sich in einen die Leibeshöhle durchsetzenden Cölomstrang (Fig. 3 und 4) fortsetzt, scheinen an den folgenden Urwirbelstielen beide Bildungen

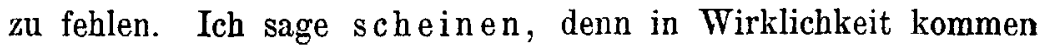
auch hier wie Fig. 5 zeigt, beim Einschneiden der Urwirbelstiele $(\mathrm{N})$ die gleichen gefässhaltigen Peritonealfalten zum Vorschein, wie in Fig. 2, nur ragen diese jetzt $n i c h t$ frei in die Leibeshöhle vor. Sie treffen vielmehr, wie aus einem Vergleich. der beiden Figuren ohne weiteres ersichtlich, auf die jetzt zur Vornierenhöhe emporgestiegene Dotterarterie (Da) und verschmelzen mit deren Überzug. So rückt also auch hier die Vene an das. Mesenchym der Dotterarterie heran, aber sie erreicht es direkt ohne Zuhilfenahme eines Cölomstrangs, weil die Dotterarterie bis nahe $\mathrm{zu}$ ihr emporgerückt ist. Damit ergibt sich eine einfache Erklärung für den Cölomstrang am vorderen Ende der Vorniere: er stellt keine Bildung sui generis dar, sondern nur eine Spezialform der auch in den übrigen Vornierensegmenten vorhandenen Verbindung der Vena cardinalis mit dem Mesenchymlager der Dotterarterie. Wo die Dotterarterie hoch stebt, kommt diese Verbindung durch direkte breite Berührung zustande, da wo sie tief steht, wie am vorderen Vornierenende, geschieht dies mittelst eines. dünnen Peritonealstranges.

Die Cölomstränge der rechten Vornierenregion stellen so. auffallende Gebilde dar, dass sie begreiflicherweise schon von früheren Untersuchern gesehen worden sind. Wir finden sie bei van Wijhe (1¿89) und bei Rab] (1896) für die rechte Seite von Pristiurusembryonen beschrieben und abgebildet. Die Schilderung van Wijhes leidet daran, dass der rechtsseitige Cölomstrang mit einer ganz anderen linksseitigen Bildung (s. S. 406. meiner vorhergehenden Arbeit) gemeinsam dargestellt wird. Doch ist er, nach den Figuren zu urteilen, mit dem von mir bei Torpedo gefundenen Strang identisch (vgl. meine Fig. 3 mit van Wijhes Fig. $6 \mathrm{f}$ ), vorausgesetzt, dass er wirklich, wie Archiv f. mikr. Anat. Bd. 89. Abt. I. 
va n Wijhe angibt, eimen Ast der Kardinalvene enthält. ${ }^{1}$ ) Dass auch ein Zweig der Aorta in den Strang eintritt, wie es van Wijhe beschreibt, habe ich bei l'orpedo nicht gefunden, doch ist es nach dem Verhalten späterer Stadien (s. unten) sehr wohl denkbar, dass bei diesem Objekt ein in Rückbildung begriffenes arterielles (unergefäss mit der Vene in einen gemeinsamen Peritonealstrang zll liegen kommen kaun. Den Strang als solchen als einen Glomus anzusprechen. wozu van Wijhe neigt, ist nicht berechtigt, doch gehen von dem Vesenchymlager, zu dem er zieht, die glomerularen Gefässbuchten und die soliden (ilomeruluszapfen aus (s. Rückert, 1916).

Der von $\mathrm{Rabl}$ abgebildete Strang stimmt ebenfalls mit dem von mir bei Torpedo gefundenen überein (vgl. Rabls Taf. 14, Fig. 5 und 6 mit meinen Fig. 4 und 9), so dass ich an der Identitït der beiderlei Bildungen keinen Augenblick zweifeln würde, wenn dem nicht die bestimmte Angabe $\mathrm{Rab} / \mathrm{s}$ im Wege stünde, dass das Gefäss des Stranges vorn mit der Aorta und hinten mit der Dotterarterie sich verbindet (?). Bei Torpedo geht es von der $V$. cardinalis aus und endet blind neben der Dotterarterie. Ist Rabls Beobachtung zutreffend, dann muss man mit der Möglichkeit rechnen, dass der Strang bei Pristiurus nicht einen Kardinalvenenast, sondern ein enges und in die Länge ausgezogenes arterielles Quergefäss führt, eine Bildung; der ich bei Torpedo nicht begegnet bin. Ein sicheres Urteil lässt sich aber ohne Kenntnis des Objektes auch hier nicht fällen. Jedenfalls haben beide Autoren die Bedeutung der von ihnen beschriebenen stränge nicht erkannt.

Für die alteren Stadien, zu denen wir jetzt übergehen, muss zuerst die Ausdehnung der Vorniere und das Verhalten ihrer arteriellen Quergefässe kurz berührt werden.

Schon bei den bisher beschriebenen Embryonen war die Abgrenzung der Vorniere nach vorn wegen der rudimentären Beschaffenheit ihres Anfangsstückes auf Querschnitten nicht ohne weiteres möglich. Nur auf horizontalen Längsschnitten war eine vollständige Übersicht über diese Organanlage. zu gewinnen. Solche Schnitte liessen zugleich erkennen, dass hinter jedem der

1) In diesem Fall hat der Strang mit dem in Fig. 18 meiner alten Arbeit (1888), mit dem ihn van Wijhe vergleicht, nichts zu tun, denn diese Figur 'zeigt nur die Dotterarterie mit einem aufsitzenden Glomeruluszapfen. 
sechs Vornierensegmente auf der rechten Seite ein arterielles buergefüss liegt. Mit Hilfe dieser Gefässe gelang es nun, das vordere Ende der Vorniere auch auf Querschnitten zu bestimmen, bei einem 'Teil der Embryonen mit Sicherheit, waihrend bei anderen infolge der mangelhaften Ausbildung des ersten und letzten Quergrefasses mit einem Irrtum um ein Segment gerechnet werden musste. Diese Fehlerquelle war auch durch die Bestimmung der somiten nicht $\mathrm{zu}$ beseitigen, weil sich die Möglichkeit einer geringen Schwankung in der Ausdehnung des Organs nicht immer ausschliessen lāsst.

Bei den nun folgenden alteren Embryonen steigert sich diese Schwierigkeit, weil die Peritonealrinne, $z u$ welcher die kranialen Vornierendivertikel schon frühzeitig verschmelzen, nunmehr weitgehend verstreicht. Man kann im allgemeinen sagen, alass dann etwa die vordere Häfte der ehemaligen Vornierenanlage infolge dieser Rückbildung nicht mehr oder doch nur sehr undeutlich zu erkennen ist, und zwar versagen jetzt nicht nur die Querschnitte, sondern auch die bisher noch zuverlässigen hori\%ontalen Längsschnitte.

Dafür geben aber wiederum die Quergefüsse, die sich auf der rechten Seite länger als die Vorniere erhalten, auch jetzt noch brauchbare Anhaltspunkte. Ich habe über ihr Verhalten und ihre Beziehungen zur Bildung der Dotterarterie in der voranstehenden Irbeit ( $R$ ückert, 1916, S. 421 und 444) neue Angaben gemacht, auf die ich hier verweise. Da aus denselben hervorgeht, dass die Vornierenquergefässe nicht zur Dotterarterie verschmelzen," sondern sich bis auf eines, das zur Wurzel der Dotterarterie wird, zurückbilden, so kann man an allen älteren Embryonen, bei welchen die Reste der sämtlichen sechs Quergefüsse noch auffindbar sind, die ehemalige Längsausdehnung der Vorniere nachträglich eruieren. Sind nur mehr fünf Quergefässe nachweisbar, dann ist wieder ein Irrtum um ein Segment nicht auszuschliessen.

Nach diesen orientierenden Vorbemerkungen verfolgen wir die Weiterentwicklung der hinteren Kardinalvene bei einem Embryo von ca. 49 Rumpfsomiten, dessen fünfte Visceraltasche in Durchbruch begriffen ist und dessen sechste das Mesoderm an ihrem linteren Umfang schon verdrängt hat.

Bei diesem Embryo ist die rechte Kardinalvene schon 
ïber die ganze Länge der Vornierenregion angelegt. In deri vorderen zwei Dritteln der Gegend bildet sie statt der bisherigen getrennten Abschnitte jetzt ein durchlaufendes Hohlgefäss, das nur einmal durch ein ganz kurzes unfertiges Stück unterbrochen ist. Auch über das hintere Drittel der Region dehnt sich ihre Anlage aus, aber sie ist hier noch grösstenteils solid und lässt auch die segmentale Anordnung vermissen, die im vorderen Abschnitt beim vorigen Stadium (Fig. 11) vorhanden war. Es schneiden jetzt fünf aufeinanderfolgende Urwirbelstiele der Vornierengegend (Nephrotome), indem sie dorso-lateral aus der Leibeshöhle empordringen, Falten der dorsalen Cölomwand für die Vene aus, wie früher in Fig. 2. Eine nähere Beschreibung ihres Verhaltens verschiebe ich auf das nächste Stadium und bemerke nur, dass hier die zweite Venenfalte des Vornierenabschnittes sich zu dem die Leibeshöhle nach hinten durchsetzenden Cölomstrang abschnürt. Dieser ist gefässlos und endet am vorderen Umfang des dritten arteriellen Quergefüsses, des einzigen zur Dotterarterie durchlaufenden.

Die linke Kardinalvene ist hier in der Entwicklung noch etwas zurück. Sie erstreckt sich nur über die vordere Hälfte des Vornierengebiets und ist hier noch dreimal unterbrochen durch ebensoviele aufeinanderfolgende Urwirbelstiele. Diese bilden die gleichen Falten wie rechts, wobei sie die Vene ventral von der Aorta abdrängen. In jeder Falte endet die Vene sofort und setzt sich nur in Form von Zellen noch weiter in sie fort. Sämtliche Falten sind hier, wie stets auf der linken Seite, an ihrem ventralen Umfang frei (s. S. 470).

Es folgt nun ein Embryo mit 57 Rumpfsomiten und sechs Visceraltaschen, von denen die erste bis fünfte weit eröffnet sind. Die Visceralbogen $2-4$ tragen die ersten knopfförmigen Anlagen äusserer Kiemen. Es sind hier noch fünf deutliche Vornierenquergefässe erhalten, zwei vordere weite, die zur Dotterarterie durchlaufen, und drei folgende rückgebildete, die blind oberbalb dieser Arterie enden.

Die Kardinalvene der rechten Seite, deren Verhalten in Fig. 12 übersichtlich dargestellt ist, dehnt sich hier über das gesamte ehemalige Vornierengebiet aus und zwar mit Ausnahme eines hinteren Endstückes als ununterbrochenes Rohr. 
Am vorderen Ende der Vornierenregion tritt die Kardinalvene wie bisher; indem sie sich ventro-median auszieht, in den Cölomstrang ein. Aber sie endet im vorliegenden Falle schon an dessen Abgangsstelle $\left(\mathrm{C}_{1}\right.$ in Fig. 12) von der dorsalen Peritonealwand und setzt sich nur mit einigen Zellen in ihn fort in Fig. 12 punktiert, so dass der Strang gefiusslos durch die Leibeshöhle zielıt. Er läuft nach linten zur ersten Wurzel der Dotterarterie zu der Stelle, wo diese von der Aorta abgeht. Der Fortschritt gegenüber den ,üngeren Embryonen besteht nun darin, dass die Kaldinalvene jetzt nicht mehr nach ibrem Eintritt in den Cölomstrang unterbrochen ist, sondern über diese Stelfe hinaus kaudal sich fortsetzt, so dass der in den Strang sich einsenkende Zipfel jetzt nicht mehr ihr Ende (wie in Fig. 11), sondern einen ventralen Seitenast ihres Stammes vorstellt (Fig. 12). In welcher Weise diese Kontinuitït der Vene nach hinten sich hergestellt hat. soll später erläutert werden.

Es folgen jetzt die zwei grossen $\{$ uergefässe, welche von der Aorta zur Dotterarterie durchlaufen. Hinter der Einmündung jeder dieser "Wu rzeln" tritt der dorsale Umfang der Dotterarterie zutage, bedeckt von seinem Mesenchymlager (Fig. 6). Auf dieses kommt nun die Kardinalvene (Cv) zu liegen, die sich gegen diese Schicht ventro-median auszieht. Sie rückt damit an das Ursprungsgebiet der Glomeruli heran, welch letztere allerdings im vorliegenden Stadium schon fast vollständig wieder zurückgebildet sind. Doch ist gerade auf dem Schnitt der Fig. 6 ein solches Gebilde noch erhalten in Gestalt eines zipfelförmigen Fortsatzes (Gl), der gegen die Vornierenrinne zu vorspringt.

Durch die auf die Quergefüsse folgenden Urwirbelstiele wird die Vene wie bisher nach unten verdrängt, sie rückt ventral von Aer Aorta $a b$ und damit auf das Mesenchymlager zu. Fig. 6 zeigt den auf das zweite Quergefäss folgenden Urwirbelstiel (N) mit seiner Höhle. Die Vene liegt ventro-lateral von ihm. Freilich kommt es hier durch das Einschneiden des Urwirbelstieles nicht zur Bildung einer frei in die Leibeshöhle herabhängenden Venenfalte der dorsalen Cölomwand, wie sie für das vordere Vornierenonde in Fig. 2 dargestellt wurde und wie sie im vorliegenderr Stadium an derselben Stelle und auch hinter dem ersten Quergefäss noch teilweise vorhanden ist, weil die Dotterarterie in der Umgebung des zweiten, mächtigsten aller Quergefässe bis zur dorsalen 
Peritonealwand empolragt. Das Verhältnis ist hier ganz das gleiche wie in Fig. $\bar{j}$ eines jüngeren Embryo. und man kann sich auch hier die freie Falte konstruieren, wenn man die laterale Wand des Urwirbelstiels um den ventralen linfang der Tene herum gegen die Vornierenrinne $z u$ in Gedanken weiter zieht.

Die dritte bis fünfe Vornielenaterie sind enge. blind geschlossene Aortenäste, die umsomehr rückgebildet erscheinen, je: weiter kaudal sie liegen. Die dritte ist in Fig. $7\left(Q_{3}\right)$ dicht hinter ihrer Abgangsstelle von der Aorta getroffen. Sie erreicht den hier schon tiefer stehenden dorsalen Umfang der Dotterarterie und damit auch dessen Mesenchymlager nicht mehr, sondern ist mit iłm durch ein schmales liekröse (()uergefässgekröse s. Rü ck er t, 1916; S. 436) verbunden. An diesen Arterienast legt sich nun die Vena cardinalis (Cv) dicht an, wobei sie sich gegen das Gekröse und das Mesenchymlager zu intsrieht.

Die vierte Vornierenarterie (ligg. i $\left(Q_{4}\right)$ ist etwas kürzer als die dritte. Auch sie besitzt ein znr Dottelarterir: ziehendes Gekröse. das sie aber nicht mehr erreicht. Die Kardinalverie, $(\mathrm{Cv})$ verhält sich ebenso wie, gegenüber der dritten Vórnierenarterie, ja, sie zieht. sich noch stäker gegen das Arteriengekröse und das Mesenchymlager zu aus.

I)a, wo die Urwirbelstiele hinter der dritten und vierten Vornierenarterie in die dorsale Cölomwand eindringen, verlagern sie wieder in der üblichen. Weise die Kardinalvene ventral, Der schnitt der Fig. 8. der den Urwirbelstiel (N) grade trifft, zeigt dies Verhalten. Infolge der Senkung des dorsalen Umfanges der Dotterarterie werden wieder freie Venenfalten durçh die Urwirbelstiele aus der Cölomwand ausgeschnitten. Die betreftenden Schnitte wurden nicht, abgebildet, doch kann.man in Fig. 8, welche das vierte Nephrotom noch im kaudalen Anschnitt zeigt, die Falte sich zur Anschauung bringen, wenn man sich wieder die laterale Yephrotomyand unterhalb der Vene. mit der dorsalen Cölomwand verbunden denkt.

Die fünfte Vornierenarterie ist wieder etwas kürzer als - die vorhergehende. Unter ilu entsendet die dorsale Colomwand nochmals ein Gekröæe zur Dotterarterie, das aber gawz rudimentär und-bereits durchrissen ist. Die Kardinalvene, die bislier die Yornierengegend kontinuierlich durchlaufen hat, tritt vor dieser Stelle nach, vorübeigehender i Unterbrechung nochmalis 
als kurzes neugebildetes Endstück auf (C'vo. Fig. 12). Es liegt hier in der Peritonealfalte des fünften Nephrotoms.?). Diese Falte schnürt sich nun mit dem Gefäss zu einem (ölomstrang ab, der frei durch den dorsalen Abschnitt der Leibeshöhle nach rorn zieht zum Vesenchymlager der hier noch weitel ventral abgerückten Dotterarterie, das ihm seinerseits einen zipfelförmigen Fortsatz zur Verbindung dorsal entgegenschickt. So gelangt der Strang an den hinteren Umfang des Gekröses des vierten Quergefässes. In Fig. 9 ist er auf seinem Weg durch die Leibeshöhle abgebildet.

Auf diese Weise kommt jetzt am hinteren Ende des Vornierenstücks der Kardinalvene das gleiche auffallende Gebilde zustande, das wir bisher nur vorne angetroffen haben. Die Ursache für die Erscheinung ist die gleiche wie dort, es ist der Tiefstand der Dotterarterie (Da, Fig. 9). Die Vene sucht hier wie in allen vorausgehenden Nephrotomen Verbindung mit denı Mesenchymlager der Dotterarterie und kann diese bei dem 'Tiefstand des hinteren Arterienumfangs nur durch einen eigenen Peritonealstrang erreichen.

Es treten also in den einzelnen Vornierensegmenten der rechten Seite sehr wechselvolle Querschnittsbilder zutage, die das Verständnis nicht nur der Kardinalvene, sondern der gesamten Vornierenregion ungemein erschweren. Sie sind bedingt durch das ungleichmässige Verhalten der einzelnen Vornierenquergefässe eines Embryo, das sich jetzt, zur \%eit ihrer Rückbildung, gegen. früher noch gesteigert hat. Aber sie lassen sich gerade unter Berücksichtigung dieses arteriellen Gefässverhaltens auf ein gemeinsames Prinzip zurückführen und damit erklären:

1. Zur Zeit der Rückbildung der Vornierenquergefässe ragt die Dotterarterie nur an den Einmündungsstellen der weiten, mit ihr in offener Verbindung gebliebenen Quergefüsse - es sind das im vorliegenden Stadium nur ein bis zwei. Stück - bis zur dorsalen Cölomwand empor. Infolgedessen senkt sich hier die Kardinalvene, wenn sie

1) Hier schnürt sich der einzige erhalten gebliebene Vornierentrichter ab. Es folgt kaudal im Bereich des sechsten Nephrotoms noch ein weiterer Trichter, der aber schon stark rückgebildet and in Abschnürung begriffen ist. Kaudal von ihm konnte ich den Überrest einer rudimentären sechsten Vornierenarterie auffinden. 
durch die auf die Quergefasse folgenden Liwirbelstiele ventral verschoben wird, direkt auf das die Dotterarterie deckende Mesenchymlager (Fig. 6). ${ }^{1}$ )

In seinem übrigen Verlaufe reicht das ehemalige Vornierenstück der Dotterarterie jetzt nicht bzw. nicht mehr bis zur dorsalen Cölomwand empor. Aber auch hier strebt die Kardinalvene dem Mesenchymlager der Dotterarterie zu und zwar auf zweierlei Weise.

2. Im Bereiche von măssig zurückgebildeten Vornierenquergefüissen, die noch durch ein Arteriengekröse mit der etwas gesenkten Dotterarterie verbunden sind, zieht sie sich gegen dieses Gekröse zu aus (Fig. 7 und 8).

3. $W_{0}$ dagegen keine solche Verbindung vorhanden ist und die Dotterarterie zugleich noch tiefer steht, zieht die Kardinalvene in einel eigenen Peritonealfalte (Cölomstrang) auf das Mesenchymlager zu. Es findet sich diese Anordnung am vorderen Ende der Vornierenregion, woselbst sie gegenüber der am Darm emporsteigenden vorderen Wand der Dotterarterie von Anfang an besteht (Fig. 3 und 4) und sie kann später auch am hinteren Ende auftreten (Fig. 9).

Wenn man von diesem Verhalten der arteriellen Gefässe ausgeht, versteht man nicht nur die wechselnden Querschnittsbilder in den einzelnen Segmenten des gleichen Embryo, sondern auch die individuellen Unterschiede, die zwischen den Embryonen der gleichen Altersstufe auftreten. Und namentlich wird man auch die Veränder'ungen richtig beurteilen, welche die mit zunehmendem Alter fortschreitende Rückbildung der Arterien bedingt. In letzter Hinsicht sei besonders darauf hingewiesen. dass die Dotterarterie ihre ursprünglich horizontale Verlaufsrichtung in der Vornierengegend allmăhlich aufgibt, indem sie von ihrer Aorten wurzel an nach hinten eine steil absteigende Richtung annimmt ( $R$ ü $c k e r t$ 1916 , S. 435). Wenn nun der hinter der Aortenwurzel gelegene Teil der Dotterarterie sich senkt und von der dorsalen Peritonealwand - entfernt, werden die anfänglich breiten Verbindungen

1) $\mathrm{Zw}$ is chen den Urwirbelstielen d. h. im Bereich der Quergefässe selbst, liegt die Vene an ihrer typischen Stelle seitlich rom ventralen Aortenumfang, also an der Abgangsstelle der Quergetässe. Dies Verhalten wurde nicht eigens abgebildet, es ist ähnlich wie in Fig. 4 und 9. 
zwischen jenem Gefäss und der dorsalen Leibeshöhlenwand an den Stellen der Quergefüsse in die Länge ausgezogen. So treten auch hier Arteriengekröse und vielleicht auch jene dünnen Cölomstränge auf, die anfänglich nur am vorderen und dann am hinteren tiefer stehenden Ende der Dotterarterie zu tinden waren. Was die letztere Möglichkeit anlangt, so sei erwähnt, dass ich bei einem Embryo am vierten Vornierensegment einen typischen, von tiner Venenfalte ausgehenden Cölomstrang gefunden habe, der aber nach abwärts frei endete. Offenbar ist er durch Zug von dem Mesenchymlager der Dotterarterie abgetrennt worden.

Nach dieser ausführlichen Darstellung kann ein zweiter gleichaltriger Embryo kürzer behandelt werden. Die Unterschiede im Verhalten der Vena cardinalis gegenüber seinem Vorgänger sind einmal dadurch bedingt, dass unter den fünf vorhandenen Quergefässen nur mehr ein einziges, weites, zwischen zwei Crwirbelstielen zur Dotterarterie durchlauft. Ausserdem zeigt die Kardinalvene noch eine unterbrochene Strecke in der Mitte ihres Vornierenabschnittes. Del" hintere ,Cölomstrang" fehlt.

Auf der linken Seite ist bei beiden Embryonen dieses Stadiums die Vena cardinalis in der hinteren Halfte des Vornierengebiets ebenfalls als Hohlgefüs aufgetreten wie rechts. Im Hereich der Urwirbelstiele liegt sie hier in den durch diese abgegrenzten Peritonealfalten, die sich inzwischen gebildet laben (Fig. 7 rechts im Bild). Dagegen sind in der vorderen Halfte der Vornierenregion diese Venenfalten wieder verloren gegangen, ${ }^{3}$ ) so dass das Gefäss von jetzt an über der ungefalteten dorsalen Peritonealwand liegt. Dieses Verhalten stelit den definitiven $\mathrm{Zu}$ stand dar. Später bilden sich auch im hinteren Abschnitt der Vornierengegend die Falten zurück und ebenso diejenigen auf der rechten Seite, soweit sie hier vorhanden waren. Diese Umgestaltung ist durch eine Rückbildung der Urwirbelstiele verursacht, die ihre die Falten bedingenden offenen Cölommündungen durch Verschluss verlieren, so dass sie dann nur mehr solide, keilförmige Zapfen del dorsalen Peritonealwand vorstellen.

Bei beiden Embryonen sind die linken Kardinalvenen an den Crwirbelstielen immer noch unterbrochen. queflacht.

1) Schon in Fig. 7 hinter der Mitte der Vorniere ist die Falte ab- 


\section{Zusammenfassung von Abschnitt 2.}

Die Vena cardinalis posterior entsteht zuerst in der Vornierengegend. Ihre Anlage beginnt hier im vorderers Abschnitt der: Region und schreitet von da langsam gegen deren hinteres Ende fort. Sie tritt diskontinuierlich auf. und zwar in dell vorderen zwei Dritteln der Vornierengegend ausgesprochen intermetamer, während. für. das hintere Drittel dies nicht nachweisbar war. Fin den intermetameren Mesenchyn: räumen aus erstrecken sich die GefäBstücke oder die sie fortsetzenden Gefässzellenketten in das Beleich des kaudal folgenden Urwirbelstiels, wo sie enden (Fig. 11). Später vereinigen sich diese-getrennten Anlagen zu eimem durchlanfenden Lai $n g s$ : gefass (lïg. 12).

Wenn die Gefassanlage - die diskontinuierliche sowie dit spätele kontinuierliche - an einen Urwirbelstiel herantritt. senkt sie sich unter ihn, indem sie von ihrem neben dem ventralen Aortenumfang gelegenen Ausgangspunkt ventral abrückt (Fig. 11 und 12).

Die vom Cölomraum aus in die dolsale Leibeshöhlenwant eindringenden Lrwirbelstiele schneiden aus ihr : Peritonealfalten atus, die frei ventral (ventro-median) in das Cölom helahhängen (Fig. 2, Fig. 7 rechts im Bild). In diesen Falten liegt die Vena cardinalis. Sie kommen links in der ganzen Vornierenregion zur Entwicklung; rechts nur da, wo die Raum $†$ verhältnisse des dorsalen Cölomabschnittes dies gestatten. Die Venenfalten sind vergängliche Gebilde. Wit der. späteren Ver:ödung der Urwirbelstiele gehen sie in der dorsalen Cölomwand auf. Damit liegt dann die Vene auch in der Vornierengegend über der geglätteten dorsalen Leibeshöhlenwand wie im übrigen Rumpfe.

Die Beziehungen der Vena cardinalis zu den Glom erulus.a $\mathrm{I}$ age $\mathrm{n}$ sind folgende: Auf der linken Seite sind die Glometrulusanlagen, die hier als ventral in die Leibeshöhle vorragend Peritonealfalten auftreten, ebenso wie die in ihnen enthaltenea (ilomerulusarterien, schon fast vollstandig zurückgebildet, wenre die Kardinalvene in ihrer Umgebung erscheint. Nur an einzelnein günstigen Stellen kaun mall erkennen, dass die Vene lateral dicht neben der Arterie in der bereits abgeflachten Glomerulusfalte sick anlegt. Wenn die Glomerulusfalte vollständig verstrichen ist; 
liegt die Vene in ihrer eigenen Peritonealfalte, der Venenfalte.

Auf der rechten Seite wird die Entstehung freier Venenfal te n dadurch eingeschränkt, dass die den dorsalen Cölomabschnitt einnehmende mächtige Dotterarterie sich streckenweise direkt an die dorsale Peritonealwand anlagert. Nur wo dies nicht der" Fall ist, nämlich an dém tiefér stehenden vorderen (Fig. 2. und. 4) und hinteren Ende der anfangs horizontal nach rückwärts verlaufenden Dotterarterie (vgl. Fig. 9), können freie Falten der dorsalen Cölomwand auftreten. Später, wenn die Arterie eine àbsteigende-Richtung annimmt, indem sié sich mit ihrem kaudalen Ende senkt, erweitert sich naturgemäss das Gebiet der Falten.

Das Verhalten der Kardinalvenenanlage an den einzelnen Stellen des rechtsseitigen Vornierengebietes ist dementsprechend ein verschiedenes:

1. Wo die 1)otterarterie sich an die dorsale l'eritonealwand direkt anlehnt, also an ihren stärker entwickelten vuergefässen, verschmelzen die I'eritonealfalten, welche die Vene umschliessen, mit dem splanchnopleuraüberzug dieses grossen Gefässes. Dadurch kommt das in die Falten herabgetretene Venenstück $d i r e k t$ a u $d$ as die Dotterarterie deckende Mesenchymlager zu liegen. Dieses let\%tere aber stellt den Mutterboden für die Glomeruli dar, von welchem einzelne bis in diese spate Entwicklungszeit sicherhalten (Fig. 6).

2. Auch da, wo die Dotterarterie nicht unmittelbar an die dorsale Peritonealwand hinaufreicht, steht sie mit ihr an der Abgangsstelle der arteriellen Quergefässe in Verbindung durch Peritonealduplikatúren, in denen die hier rückgebildeten Quergefässe verlaufen oder denen sie zustreben. In diese Arteriengekröse zieht.isich die Kardinalvene ventral aus, indem si.e dicht lateral von der Arterie dem Mesenchymlager zustrebt (Fig. 7 und 8).

3. Und selbst da, wo die Quergefässe stark rudimentär sind und keine Verbindung durch Arteriengekrose zwischen der dorsalen' Peritonealwand urd 
der tief stehenden Dotterarterie vorhanden ist, verläuft die Vene oder ein sie fortsetzender Gefässzellenzug frei. nur von einem Peritonealüberzug umhüllt, als cölomstrang durch die Leibeshöble zum Mesenchymlager der Dotterarterie. Dieser Strang ist am vorderen Ende der Vornierenregion vom Beginne der Venenbildungen an regelmässig zu finden (Fig. 2 und 4.) Später tritt er auch am hinteren Ende inkonstant auf (Fig. 9) und kommt in noch spüterer Zeit wie es scheint auch $\%$ wischen dieser Stelle und der Dotterarterienwurzel vor.

Las hiel zusammengefasste lerbalten gestat tet die Deutung, dass die ersten, metamer oder richtiger intermetamer auftretenden Anlagestücke der hinteren Kardinalvene der Vornierengegend rudimentäre, verspatet a uftretende clomerulusvenen sind (Fig. 11). Auch das spater durch Verschmelzung derselben entstandene durchlaufende venenroli halt durch segmentale Ausbiegungen gegen das Mesenchymlager der. Dotterarterieseille Beziehungen zum Glomerulargebiet nocheine\%eitlanganfrecht (Fig. 12).

\section{Weitere Entwicklung der Kardinalvene.}

Die vorstehende Auffassung erfährt eine wesentliche Stütze, wenn man die Entwicklung der hinteren Kardinalvene im Bereich der Vorniere weiter verfolgt und mit deren Entstehung im übrigen Rumpfgebiet vergleicht. Dies soll im Folgenden geschehen.

Bei dem Embryo unseres vorletzten Stadiums (49 Rumpfsomiten), bei welchem die Vene in der hinteren Halfte der Vornierenregion als unterbrochene und unfertige Anlage aufgetreten war, reicht sie nicht über dieses Gebiet nach hinten hinaus, wenn ich yon ein paar vereinzelten Schnitten absehe, auf welchen ich ihre solide Anlage, und einmal auch ein kleines Endothelrohr, wahrzunehmen glaube. Anders in unserem letzten Stadium (5.7 Rumpfsomiten). Hier, wo die Venenanlàge sich in der hinteren Halfte der Vorniere weiter ausgebildet hat, überschreitet sie schon diese Region nicht unbeträchtlich. Bei dem einen Embryo über sechs, bei dem anderen um zwölf Segmente. Ihre Entwicklung ist also im Vergleich zu der des Fornierenstückes rasch 
nach hinten vorgeschritten. Sie ist auf dieser Strecke allerdings enger als davor im Vornierengebiet und zeigt stärkere Unterbrecliungen, die namentlich nach hinten. wo die Anlage ganz jung und unfertig erscheint, an Länge zunehmen.

Es folgen zwei Embryonen eines noch alteren bisher nicht berïcksichtigten Stadiums, bei welchen die sechste Kiementasche durchgebrochen, die ausseren Kiemen schon als kurze Fädchen, und der solide Oesophagus mit rundlichem, noch nicht in die Breite ausgezogenem Querschnitt aufgetreten sind. Die Zahl der Rumpfsomiten wurde bei dem einen der zwei Schwesterembryonen dieses Stadiums auf 68 bestimmt. Bei dem einen dieser Embryonen erstreckt sich die Kardinalvenenanlage schon um 17 Segmente, bei dem anderen in horizontale Längsschnitte zerlegten Embryo um 15 Segmente über die Vorniere kaudal hinaus.

Hier wie in dem vorausgegangenen Stadium lässt sich deutlich verfolgen, dass die Kardinalvene ihren Entstehungsmodus aus getrennten Stücken auch kaudal von der Vorniere beibehält. Die Einzelabschnitte erscheinen aber nicht in den intermetameren Mesenchymräumen, um sich von da in das Bereich des nächsten Urwirbelstiels auszubreiten, wie das im vorderen Abschnitt der Vornierengegend der Fall war (Fig. 11), sondern sie treten sowohl im Bereich der Nephrotome auf, als auch zwischen ihnen und sind von ungleicher Lange, so dass man bei Verfolgung der Querschnittsserie den Eindruck einer ganz unregelmässigen Anordnung erhält. Horizontale Längsschnitte aber (s. Textfigur auf nächster Seite, die eine grössere Strecke der Gefässanlage überblicken lassen als Querschnitte, zeigen doch, dass auch hier eine ziemlich regelmässige, annähernd segmentale Anordnung eingehalten wird, wenn dieselbe auch durch die ungleiche Längenausdehnung der Einzelstücke streckenweise gestört erscheint. So breitet sich eines der Venensegmente über zwei Urwirbelstiele aus, wobei es offen bleiben muss, ob es nicht vorher aus zwei Segmenten entstanden ist.

Auf dieser hinter der Vorniere gelegenen langen Strecke nimmt nun die Kardinalvene eine andere Lage zu den Urwirbelstielen ein, als im Bereich der Vorniere. Wie aus der obigen Beschreibung und aus den Figuren 2, 5, 6 und $8 \mathrm{zu}$ ersehen war, liegt sie im Vornierengebiet ventral oder genauer ventro-lateral von den Urwirbelstielen. Dabei ist sie aus ihrer als typisch anzusehenden Stellung neben dem ventralen 
Umfang der Aorta nach unten mehr oder weniger weit abgerückt. Im Gegensatz hierzu verläuft die Vene $\mathrm{kaudal}$ von der Fornie re, wenn sie auf die Urwirbelstiele trifft, d o rs al oder genauer dorso-median über diese weg, wie Fig. $10 \mathrm{C}$ zeigt. Die Urwirbelstiele, die hier die Urnierenkanălchen liefern, schmiegen

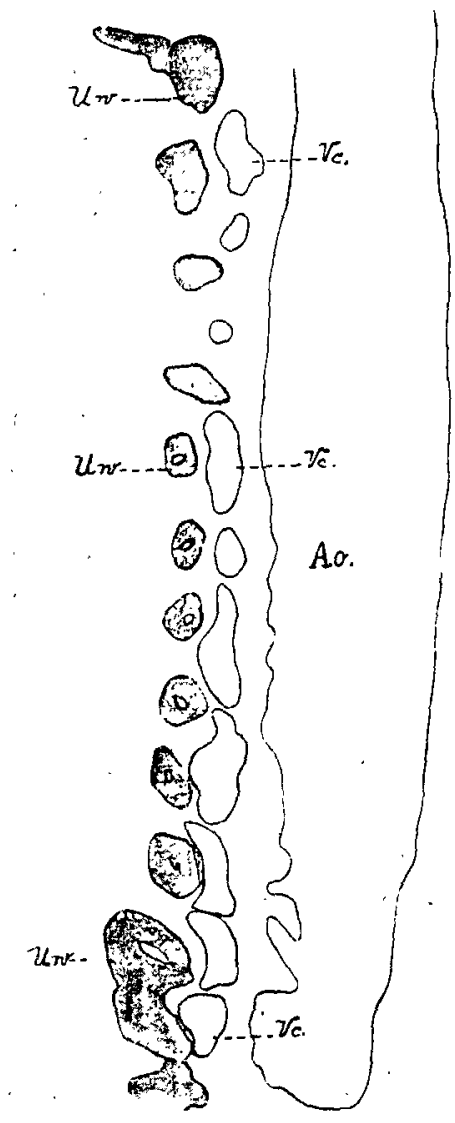

snlage der hinteren Kardinalvene kandal von der Vornierenregion. Kombination von zwei aufeinanderfolgenden horizontalen Längsschnitten durch einen Torpedoembryo von 68 Rumpfsomiten. $\mathrm{Uw}=$ Urwirbel, Vc $=$ Anlagestiicke der Vena cardinal. poster, ¿. $\quad \ldots$ A $\alpha=$ Aorta: sich dem Vornierengang dicht an, so dass zwischen beiden ein Raum für eine V'enenanlage nicht frei ist. Es kommt daber auch keine I'eritonealfalte (Venenfalte), wie sie weiter vorn zwischen der Vorniere und den stielen erscheint, zustande, sondern das unter dem Vornierengang gelegene Peritoneum biegt direkt nach aufwitrts in die laterale Wand des Trwirbelstieles um. Der Übergang zu diesem Verhalten macht sich, wie nebenbei erwähnt sei, schon im hinteren Vornierenabscbnitt bemerkbar. Das vorletzte Nephrotom riickt bereits nither an die Vorniere heran, so dass seine Venenfalte erheblich schmäler wird und am letzten Nephrotom kann man schon eine Anordnung finden, die fast derjenigen der Fig. 10 gleicht.

Gegenüber diesem eigentümlichen Verbalten muss man sich die Frage vorlegen, ob die in der Vornierengegend unter die Urwirbelstiele tretende Vene iiberhaupt der Kardinalvene des übrigen Rumpfes entspricht? Eine Antwort hierauf können wir nur erhalten, wenn wir diese abweichende Venenanlage der Vornierengegend in ibrer Weiterentwicklung verfolgen und zusehen, ob:aus ihr noch eine echte Kardinalvene wird: 
Das letztere ist nun in der Tat der Fall. Schon im Stadium anserer Fig. $6-10$ ist im Vornierengebiet die Anlage eines $z$ weiten Venenzuges dorsal von den Crwirbelstielen aufgetreten, also eines Gefässes, das in bezug auf seine Lage mit der echten Kardinalvene übereinstimmt. In Fig. 6 und 8 (C. d.) ist es auf der rechten (links) und in Fig. 7 auf der linken (rechts) Seite des Embryo in seiner Lage zum Urwirbelstiel dargestellt.

Diese dorsale Gefässanlage ist im Bereich der Urwirbelstiele voll der ventralen vollständig getrennt. In den intermetameren $Z$ wischenräumen dagegen kontluieren beide miteinander. In Fig. 7, anf der rechten Seite des Embryo, berühren sie sich, um auf dem nächsten nicht abgebildeten Schnitt sich ineinander $z u$ eröffnen. Die intermetameren Venenstücke, von welchen die dorsalen Anlagen sich abzweigen (Fig. 6 linke, Fig. 7 rechte Seite des Embryo), sind gemäss ihrer frühzeitigen Entstehung weiter als die dorsal und ventral von den Urwirbelstielen gelegenen Abschnittte (Fig. 6 rechte, Fig. 7 linke Seite des Embryo).

Einen guten Überblick über die dorsalen Gefässanlagen gibt Fig. 12. Man erkennt hier, dass sie über die zwischen ihnen gelegenen Urwirbelstiele hinweg einander zustreben. Über dem «weiten Urwirbelstiel dieser Abbildung bilden sie schon ein einheitliches Gefässrohr (während das ventrale Rohr daselbst keine Verbindung nach hinten erhalten hat), über den anderen ist die Anlage noch diskontinuierlich.

Diesen Entwicklungsgang in einzelnen hier zu schildern. unterlasse ich, schon wegen der individuellen Varianten in der jeweiligen Ausbildung der beiden Venenzüge. Doch sei hier erwähnt, dass die dorsale Anlage nach aufwärts mit Gefässanlagen in Verbindung steht, die über die Ebene der Kardinalvene hinausreichen bis in die Höhe der Chorda dorsalis (Fig. 7 rechts). Dies Verhalten stimmt mit dem hinter der Vorniere gelegenen Abschnitt der Kardinalvene überein, so dass die dorsale Gefässanlage des Vornierengebietes auch in dieser Hinsicht sich als echte Kardinalvene erweist. Inwieweit seine Entstehung mit der Bildung dieser anscheinend segmentalen Äste zusammenhängt, bedürfte einer näheren Untersuchung. Die Beziehung der Kardinalvenenbildung zu denselben ist, wie aus der Literaturübersicht hervorgeht, schon von früheren Autoren gesehen oder vermutet worden.

Was die weitere Entwicklung anlangt, so ist schon im Stadium von 68 Rumpfsomiten die dorsale Venenanlage, wie Fig. 13 zeigt, bis auf eine über dem ersten Urwirbelstiel der Abbildung noch vorbandene Unterbrechung als durchlaufendes Rohr ausgebildet. Dasselbe nimmt nach hinten (nach rechts in 
der Figur) am stărksten zu. Das ventrale dagegen wird umgekehrt gegen das hintere Ende der Vornierenregion eng und schliesslich undurchgängig. Ein Vergleich mit Fig. 12 erweckt den Anschein, dass dieses letztere Stück schon wieder der Rückbildung anheim gefallen sei. Doch vermute ich mit Rücksicht auf spätere Stadien, dass es bei dem vorliegenden Objekt nur in seiner Entwicklung zurückgeblieben ist.

Es sind also jetzt in der Vornierengegend $z w e i$ längsverlaufende Venenz üge, einventraler und dorsaler, vorhanden, die intermetamer, zwiscben den Urwirbelstielen, konfluieren, während sie im Bereich der letzteren sich trennen, um sie zu umfliessen. Diese regelmässige Anordnung wird nun dadurch gestört, dass die dorsale Vene in ihrer vorderen Hälfte erheblich schwächer als die ventrale, einmal sogar unterbrochen ist, während die ventrale umgekehrt nach hinten $z u$ in der Entwicklung zurücksteht und schliesslich durch die hier stärkere, dorsale ganz ersetzt wird. Durch das letztere Verhalten ist ein allmählicher Übergang zu der kaudal von der Vorniere gelegenen typischen, nur aus den dorsalen Anlagen aufgebauten, Cardinalis gegeben.

Die Bezeichnungen "dorsal" und "ventral" haben jetzt für das Anfangsstück der beiden Venenzüge keine Berechtigung mehr. Die Urwirbelstiele, die ursprünglich eine fast horizontale, nur wenig aufsteigende Richtung von der dorsalen Cölomwand zum Myotom eingeschlagen und damit eine dorso-mediane und ventrolaterale Gefässanlage getrennt hatten, sind jetzt im vorderen Abschnitt der Region bis auf ihr ventrales ehemaliges Mündungsstück aufgelöst. Sie bilden nur mehr kurze Stümpfe, die sich von der dorsalen Peritonealwand $n$ ach a f f a r ts erheben, sich zwischen die beiden Gefăsse einschieben, so dass diese jetzt medial und lateral von ihnen liegen.

Da diese Umgestaltung in kranio-kaudaler Richtung vor sich geht, so trifft man in späterer Zeit die beiden Gefässe über die ganze Ausdehnung der Vornierenregion in der neuen Stellung zueinander. Diese Verlagerung wird zugleich dadurch bedingt, dass durch die zunehmende Verbreiterung der dorsalen Cölomwand die beiden Gefässe nebeneinander den nötigen Platz finden. Dementsprechend zieht sich schliesslich die gesamte Venenanlage 
erheblich in die Breite aus. Von oben senkt sich dann der inzwischen entstandene Interrenalkörper in sie ein, so dass dessen ventraler Umfang rom Blut der Vena cardinalis direkt. umspült wird. Diese Beziehung der Cardinalis zum Interrenalkörper tritt von jetzt an so in den Vordergrund, dass die Vene geradezu als das abführende Gefäss dieser Drüse erscheint.

Um das Endresultat des Entwicklungsvorganges zu ermitteln, muss man die Untersuchung auf erlieblich altere Embryonen ausdehnen. Bei einem Torpedoembryo von 12,5 mm Körperlänge, dessen solider Oesophagus in die Breite ausgezogen ist, macht die Kardinalvene der Vornierengegend, wie Fig. 14 zeigt, noch mehr als im vorhergehenden Stadium (Fig. 13) einen einheitlichen Eindruck, weil sie sich wiederum ausgedehnt hat und weil die Urwirbelstiele gleichzeitig durch weitere Rückbildung kleiner geworden sind. Sie werden wie Inseln rom Venenstrom umtossen. Immer noch erkennt man deutlich, wie der kraniale Abschnitt des Gefässes ganz überwiegend durch die ventrale (laterale), der kaudale Abschnitt dagegen durch die dorsale (mediale) Anlage, die danı allein nach hinten erhalten bleibt, entstanden ist.

Erst bei einem Embryo von $15 \mathrm{~mm}$ Länge, dessen grösste Breite im Bereich der Flossenanlagen auf Schnitten 2, $5 \mathrm{~mm}$ betragt, sind die trennenden Urwirbelstiele in mindestens der vorderen Hälfte des ehemaligen Vornierengebietes ganz verschwunden, womit daselbst eine vollkommen einueitliche Kardinalvene hergestellt ist. Derjenige Urwirbelstiel, welcher die Dotterarterie kaudal begrenzt, ${ }^{1}$ ) ist zu dieser Zeit gerade noch als leichte-

1) Es folgen also in diesem Stadium auf die Wurzel der Dotterarterie. zunächst zwei rückgebildete Urwirbelstiele, von denen der vordere, der dieses. Quergefäss kaudal begrenzt, nur mehr eine peritoneale Einkerbung bildet. Diese Urwirbelstiele entsprechen dem dritten und vierten, event. dem vierten. und fünften Nephrotom, wie aus der Lage dieses Segments zu den Quergefässen in früheren Stadien mit Sicherheit zu bestimmen ist. Weiter kaudal schliessen sich an sie zwei weitere Urwirbelstiele an, die ihren epithelialen Bau beibehalten haben und an ihrem dorsalen Ende zu kleinen rundlichen, mit engem Lumen versehenen Bläschen sich erweitert haben. Sie geben sich dadurch als Arilagen von Urnierenkanälchen zu erkennen. Daraus geht hervor, dass mindestens das sechste, vielleicht aber auch das fünfte Nephrotom, also Segmente, die in ihren weiter dorsal gelegenen Abschitten vorber am Aufbau der Vorniere Teil genommen hatten, Anlagen von UrnierenkanäArchiv f. mikr. Anat. Bd. 89. Abt. I 
l'eritonealkerbe kenntlich. Dann folgt nach rückwäts ein ebenfalls, aber doch weniger stark rückgebildeter Urwirbelstiel. $\mathrm{Er}$ dringt als erster in die Vene von unten ein und trennt ebenso wie die folgenden Urwirbelteile einen kleineren lateralen Venenzug von dem grossen Hauptgefäss ab. Das laterale Gefäss ist also in der hinteren Halfte der Vornierenregion noch erbalten, doch ist es gegenüber dem medialen in der Entwicklung zurückgeblieben und verengt sich zunehmend in kaudaler Richtung. Trotzdem setzt es sich, noch enger werdend, jetzt auch über die Vornierengegend kaudal eine Strecke weit fort, indem es sich als kleine Seitenbucht diskontinuierlich vom Hauptgefäss abtrennt.

Bei einem erheblich alteren. Embryo von $22 \mathrm{~mm}$ Körperlänge und einer grössten Querschnittsbreite von $4 \mathrm{~mm}$ ist die kleine laterale Vene immer noch neben der medialen vorhanden.

Erst bei einem Embryo von über $24 \mathrm{~mm}$ Lange und $7 \mathrm{~mm}$ Querschnittsbreite auf dem Schnitt ${ }^{1}$ ) vermisse ich dieses Nebengefäss im kaudalen Teil des ehemaligen Vornierengebietes und weiter nach rückwärts. $\mathrm{Ob}$ es zugrunde gegangen ist oder schliesslich noch der Cardinalis einverleibt wurde, kann ich nicht entscheiden.

\section{Zusammenfassung von Abschnitt 4 .}

Uie Vena cardinalis posterior entsteht in dem $\mathrm{kaudal}$ von der Vorniere gelegenen längeren Abschnitt des Rumpfes ebenfalls in kranio-kaudaler Richtung und aus getrennten St ücken, die sich nachträglich zu einem kontinuierlichen Längszug vereinigen. Diese anfänglichen Einzelstücke treten aber nicht intermetamer, d. h. zwischen den Urwirbelstielen (Nephrotomen) auf, sondern sowohl im Bereich der Urwirbelstiele als $z$ wischen ihnen. Indessen halten sie, von einzelnen durch ihre

chen liefern. Dieses Verhalten, das mit den durch van Wijhe und $R$ abl bei Squaliden gemachten Beobachtungen übereinstimmt, möchte ich bei dieser Gelegenheit hervorheben, angesichts des Umstandes, dass die von mir (1888) und ran Wijhe (1889) schon vor langer Zeit vertretene Anschauung von der Nicht-Homologie der Vor- und Urniere immer wieder angezweifelt wird. Es dürfte ein vergebliches Bemühen sein, durch negative, bei anderen Wirbeltieren gemachte Befunde den klaren und positiven Tatsachenbestand bei Selachiern entkräften zu wollen.

1) Am frisch fixierten Objekt wurden von Herrn Prof. Marcus 9 mm gemessen. 
ungleiche Länge bedingten Abweichungen abgesehen, i $\mathrm{m}$ grosse $\mathrm{n}$ und ganzen eine segmentale Anordnung ein (vgl. lextfigur S. 486).

In seiner Lage $z$ u den Urwirbelstielen aber unterscheidet sich dieser kaudal von der Vorniere gelegene Abschnitt der Kardinalvene wesentlich von demjenigen der Vornierengegend, insofern er dorso-median von diesen Somitenstücken auftritt (Fig. 10) und später rein median von ihnen bzw. den aus ihnen hervorgegangenen Urnierentrichtern verläuft.

Im Gegensatz hierzu liegt in nerhalb der Vornierengegend die erste Anlage der Kardinalvene ventro-lateral (Fig. 3 und $5 \mathrm{Cv}$ und Fig. 11) und später rein lateral von den Urwirbelstielen. Alsbald aber gesellt sich im Vornierenbereich zu diesem primären Venenzug ein $z$ weiter Parallel$\mathrm{zug}$ hin $\mathrm{zu}$, der sich von ihm in den $\mathrm{zwischen}$ den Urwirbelstielen befindlichen Interstitien abzweigt und dorso-median über die Stiele hinwegläuft (Fig. 6 und $8 \mathrm{Cd}$ und Fig. 12). Dieser dorsomed i a ne Yenenzug nimmt also zu den Urwirbelstielen die gleiche Lage ein, welche die typische Kardinalvene in dem langen, kaudal auf die Vorniere folgenden Rumpfgebiet inne hat. Fr breitet sich, in kaudaler Richtung über die Vorniere hinausgreifend, rasch auf den übrigen Rumpfabschnitt aus, und stellt daselbst für sich allein die definitive Kardinalvene her (Fig. 12 und hinteres Ende der Fig. 13 und 14).

Innerhalb der Vornierenregion dagegen bildet sich die definitive Kardinalvene - sehr langsam - durch Verschmelzung der beiden die Urwirbelstiele ventral und dorsal umfliessenden Venenzüge unter allmählicher Rückbildung der sie trennenden Stiele. Dabei liefert im kranialen Abschnitt der Region die ursprünglicbe ventroIaterale (primäre) Venenbahn den grösseren Anteil der definitiven Kardinalvene, wăhrend umgekehrt im kaudalen Abschnitt diese Rolle der ursprünglich dorso-medialen Venenbahn zufällt (Fig. 13 and 14).

Es geht hieraus hervor, dass in der Vornierengegend $\mathrm{nur}$ der später entstehende dorsale Venenzug nebst den $z$ wischen den Urwirbelstielen gelegenen ersten Anlagestücken der typischen Kardinalvene entspricht. 
und dass der vorher auftretende ventrale Anteil der. Gefässanlage eine Bildung sui generis darstellt, die der Vornierenregion eigentümlich ist. Esstimm t dies zu unserer oben (S. 484) vorgetragenen Auffassung, nach welcherdiesen Venenanlagen dieBedeutung von rudimentalen Glomerularvenen zulommt. Der in der Vornierengegend zuerst auftretende venöse Längszug ist daher als eine Vornierenvene anzusprechen, die sich nachträglich dulch Hinzutreteneineszweiten Langszugesindie echte Kardinalvene umwandelt.

\section{Literaturverzeichnis.}

1909. Evans: On the develupment of the Aortes, Cardinal- and Umbilical veins and the other Blood-Vessels of Vertebrate Embryos from Capillaries. The Anat. Record, Vol. III, 1909.

1905. Grafe: Beiträge zur Entwicklung der Urniere und ihrer Gefüsse beim Hühnchen. Arch. f. mikr. Anat., Bd. 67, 1905.

1893, a. Hoffmann, C. K.: Zur Entwicklungsgeschichte des Herzens und der Blutgefässe bei den Selachiern. Morph. Jahrb., Bd. 19, Heft 4, 1893.

1893, b. Derselbe: Zur Entwicklungsgeschichte des Venensystems bei den Selachiern. Morph. Jahrb., Bd. 20, Heft 3, 1893.

1911. v. Mö̈llendorf: Über die Entwicklung der Darmarterie und des Vornierenglomerulus bei Bombinator. Morph. Jahrb., Bd. 43, 1911.

1892. Rabl, C.: Über die Entwicklung des Venensystems der Selachier. Festschrift zum 70. Geburtstage Rudolf Leuckarts. Leipzig 1892.

1896. Derselbe: Über die Entwicklung des Urogenitalsystems der Selachier. Theorie des Mesoderms, III. Morph. Jahrb., Bd. 24, 1896.

1888 Rückert: Über die Entstehung der Exkretionsorgane bei Selachiern. Arch. f. Anat. u. Physiol.. Anat. Abt., 1888.

1916. Derselbe: Über die Glomeruli der Vorniere von Torpedo und deren arterieile Gefässe. Arch. f. mikr. Anat, Bd. 89, Heft 4, 1916.

1889. Van Wijhe: Über die Mesodermsegmente des Rumpfes und die Entwicklung des Exliretionssystems bei Selachiern. Arch. f. mikr. Anat., Bd. 33, 1889. 


\section{Figurenerklärung.}

\section{Erläuterung der Bezeichnungen.}

Ao $=$ Aorta

$A g=$ Arteriengekröse (Gekröse der arteriellen Quergefässe der Vorniere).

$\mathrm{C}^{\prime}=$ Vena cardinalis kaudal von der Vornierengegend (Fig. 10)

$\mathrm{C}=\because \pi$ in der Vornierengegend zwischen den Urwirbelstielen

$\mathrm{Cd}=n \quad, \quad$ in der Vornierengegend dorsal von den Urwirbelstielen

$\mathrm{Cv}=, \quad, \quad$ in der Vornierengegend ventral von den Urwirbel-

$\mathrm{Ch}=$ Chorda dorsalis

$\mathrm{Cl}=$ Cölom

$\mathrm{D}=\mathrm{Darm}$

$\mathrm{Da}=$ Dotterarterie

$\mathrm{GI}=$ Glomeruius

Ml = Mesenchymlager auf der Dotterarterie

$\mathrm{Ns}=$ Mesenterium

$\mathrm{N}=$ Urwirbelstiel (Nephrotom)

$\mathrm{P}=$ Vorniere

$\mathrm{Pg}=$ Vornierengany

$\mathrm{Q}=$ Arterielles Quergefäss der Vorniere

$\mathrm{Sp}=$ Somatopleura

Sp. p = Splanchnopleura

$\mathrm{St}=$ Cölomstrang

Sämtliche Figuren stammen von Embryonen von Torpedo ocellata. Vergrïsserung der Schnittbilder Fig. 1-10: 210:1.

\section{Tafel XXV.}

Fig. 1. Querschnitt durch das vordere Ende der Vornierenregion von einem Torpednembryo mit $\check{\partial}$ Visceraltaschen, erste bis vierte eröffnet. 43-44 Rumpfsomiten.

Fig. 2. Querschnitt durch den gleichen Embryo, 4 Schnitte kaudal von Fig. 1.

Fig. 3. Querschnitt durch den gleichen Embryo, 3 Schnitte kaudal von Fig. 2.

Fig. 4. Querschnitt durch das vordere Ende der Vornierenregion eines wenig älteren Embryos als Fig. 1-3. Erste bis vierte Visceraltasche eröffnet, erste Anlage der sechsten Visceraltasche.

Fig. 5. Querschnitt durch die Mitte der Vornierenregion (Gegend des dritten Quergefässes) des Embryo der Fig. 4.

Fig. 6. Querschnitt durch die Vornierenregion eines Torpedoembryo mit 6 Visceraltaschen, erste bis fünfte eröffnet. 57 Rumpfsomiten. Der Schnitt streift das dritte Vornierenquergefäss im kranialen Anschnitt. Das Gefäss Cd vom nächsten Schnitt eingetragen. 
494 J. R ï ckert: Zur Entwickiung der Vena cardinalis posterior.

\section{Tafel XXVI.}

Fig. 7. Querschnitt durch den gleichen Embryo wie Fig. 6. 2 Schnitte kaudal von Fig. 6. Drittes Vornierenquergefäss getroffen.

Fig. 8. Querschnitt durch den Embryo von Fig. 6 und 7. Vier Schnitte kaudal von Fig. 7. Das vierte Quergefäss vom nächsten Schnitt eingetragen.

Figr. 9. Querschnitt durch den Embryo der Fig. 6-8. 5 Schnitte kaudal von Fig. 8.

Fig. 10. Querschnitt durch den Embryo der Fig. 6-9, 21 Schnitte kaudal von Fig. 9. Zweites Nephrotom kaudal von der Vorniere.

Fig. 11. Erste Anlage der V. cardinal. post. in der Vornierengegend des Torpedoembryo der Fig. 4 and 5 . Nach einer graphischen Rekonstruktion.

Fig. 12. Weitere Entwicklung der V. card. post. in der Vornierengegend des Torpedoembryo der Fig. 6-9. Nach einer graphischen Rekonstruktion.

Fig. 13. Dasselbe von einem Torpedoembryo mit eröffneter sechster Visceraltasche und kurzen äusseren Kiemenfäden, 68 Rumpfsomiten.

Fig. 14. Dasselbe von einem Torpedoembryo von $12,5 \mathrm{~mm}$ Körperiänge. 


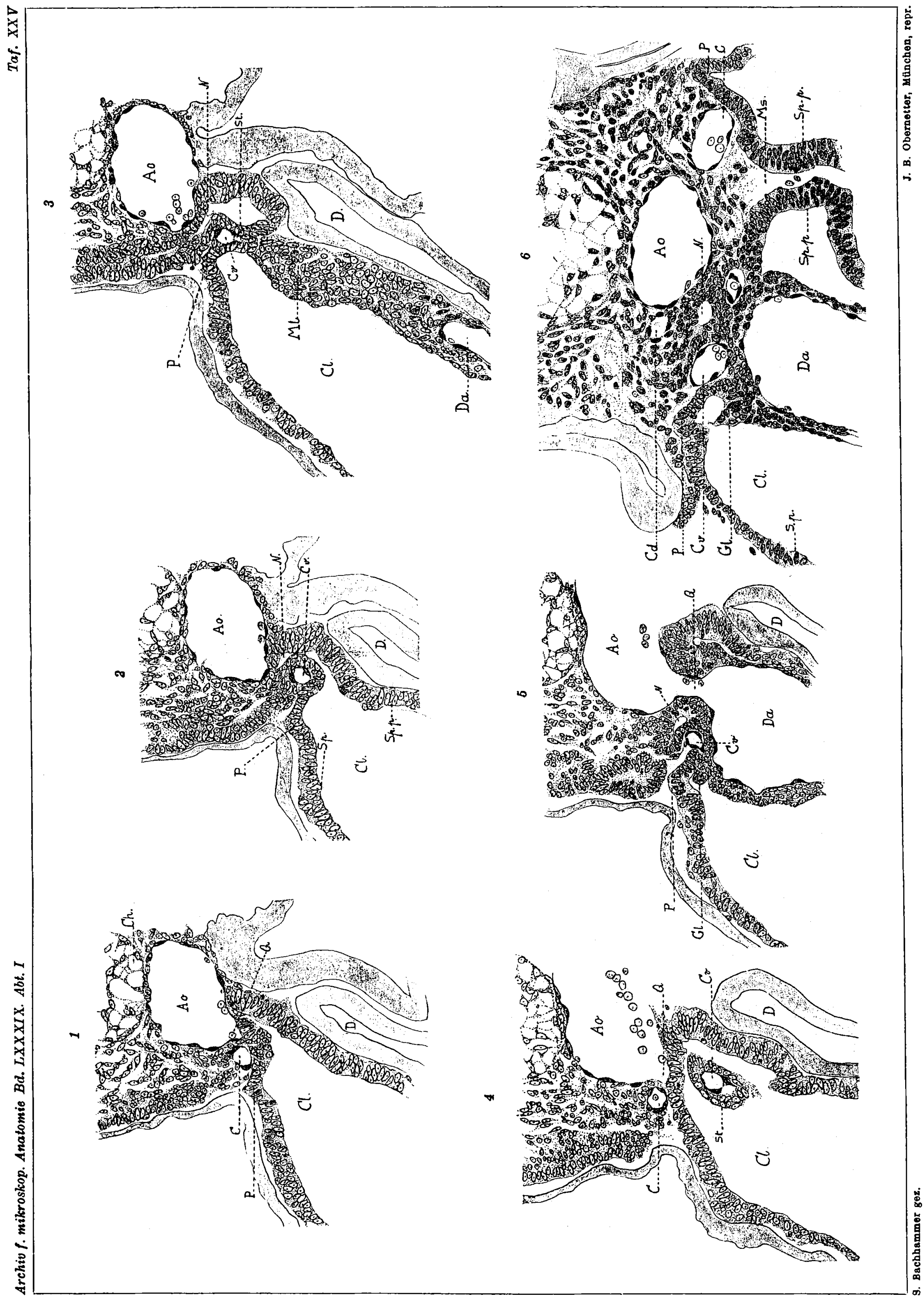




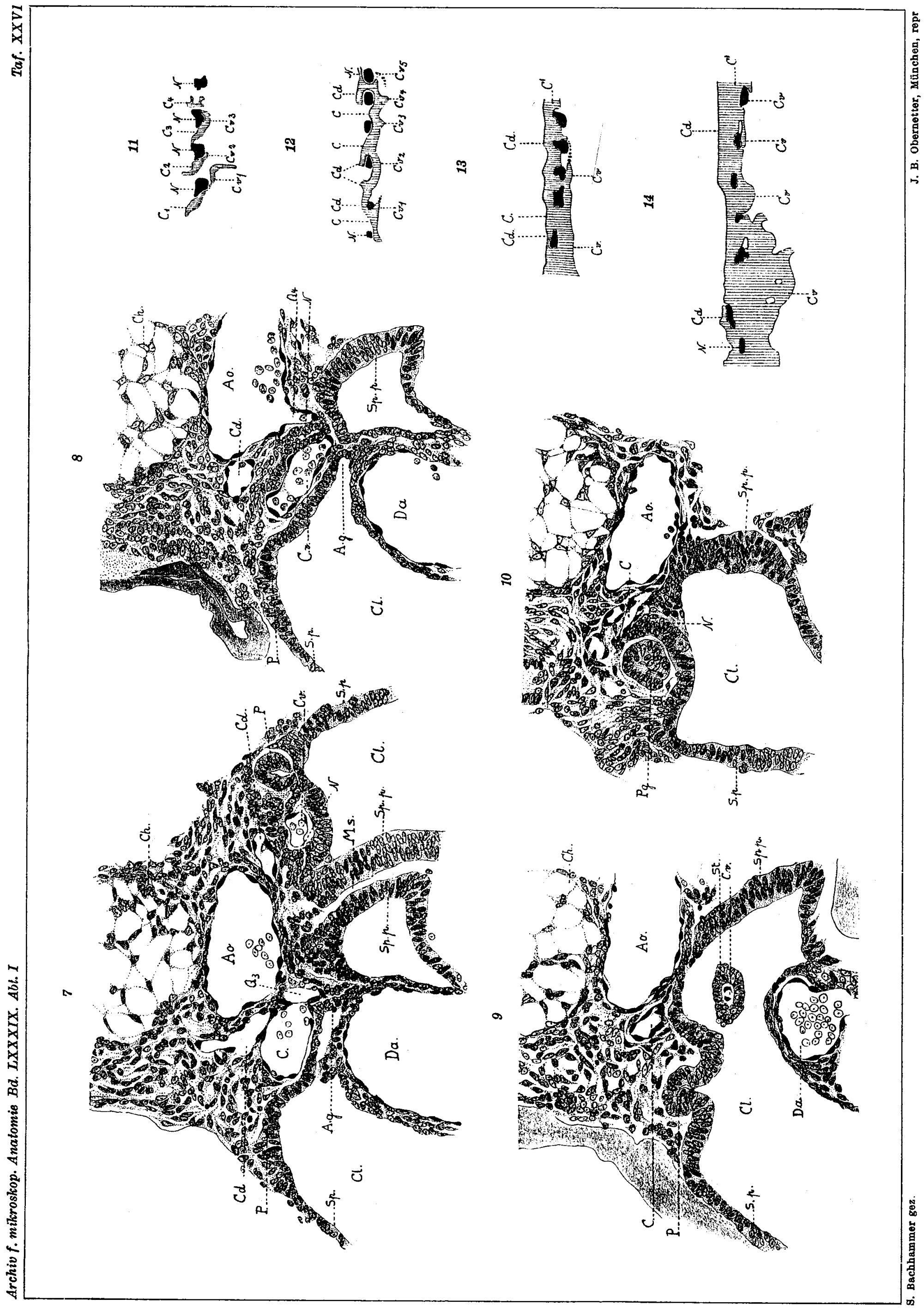

